\title{
Dentinogenesis imperfecta type II: Ultrastructure of teeth in sagittal sections
}

\author{
Aneta Wieczorek, Jolanta Loster
}

Prosthetic Department, Dental Institute, Jagiellonian University, Krakow, Poland

\begin{abstract}
The morphological abnormalities of the teeth of patients affected by dentinogenesis imperfecta type 2 (DI-II) may underlie the difficulties with the clinical restoration of such teeth. We therefore performed a scanning electron microscopy (SEM) study of four permanent first mandibular molars of four DI-II patients with periapical pathosis. The teeth were prepared for SEM evaluation by standard methods. In the crown, the enamel presented a highly irregular surface with a number of cracks and crevices. In some places, only granular remains of the enamel were found, while in other parts of the crown, the enamel was absent. SEM examination revealed the structural changes responsible for the lower enamel's hardness and resistance to attrition, and for tooth wear, while the structural changes in the dentin may explain the failure of some adhesive restorative materials. This SEM study thus revealed structural defects which underlie the problems of attrition and restoration loss found in patients with this genetic dental condition. (Folia Histochemica et Cytobiologica 2013, Vol. 51, No. 3, 244-247)
\end{abstract}

Key words: dentinogenesis imperfecta, tooth structure, enamel, dentin, SEM

\section{Introduction}

Dentinogenesis imperfecta - a developmental disorder of dentin occurring during the formation of its organic matrix - is the most common dental genetic disease, with a reported incidence of from 1 in 6000 to 1 in 8000 [1]. In 1973, Shields et al. classified this disorder into three subtypes: type I occurs in patients with the osteogenesis imperfecta syndrome; type II (DI-II) occurs in patients with dentition abnormalities only; and type III (also called "Brandywine") which occurs in isolation in a triracial southern Maryland population [2]. Clinically, affected teeth have an opalescent color, ranging from grey to brownish blue [3], due to deposits of pigments and minerals in the interior of the dentinal tubules [4]. Radiologically, the teeth are characterized by bulbous crowns, a marked cervical stricture, and short, thin roots [5]. The pulp chambers and root canals show increased odontoblastic activity, which may obliterate the dentinal tubules and pulp chambers. These anomalies of the tooth root occur

Correspondence address: A. Wieczorek,

Prosthetic Department, Jagiellonian University,

Krakow, Poland;

e-mail: aneta.wieczorek@uj.edu.pl even with incomplete symptoms of the disease. There are no radiologically visible abnormalities in the periodontium or alveolar bone [6]. Another characteristic of the condition is the absence of caries $[3,5,7,8]$.

Dentinogenesis is the process of dentin formation by odontoblastic extracellular collagen fibers, which form a network for the deposition of hydroxyapatite crystals. Noncollagenous matrix proteins (NCPs) are thought to play a major role in dentin mineralization. The major NCPs in dentin are dentin phosphoprotein (DPP) and dentin sialoprotein (DSP) [9]. The genes responsible for producing both DPP and DSP are located at 4q12-21. Disturbances in the secretion of DSP and DPP, and thus in the proper shape and placement of the crystals of apatite in the dentin matrix, are manifested clinically as dentinogenesis imperfecta. Types II and III of dentinogenesis imperfecta appear to result from mutations in the gene encoding dentine sialophosphoprotein (DSPP), suggesting that these conditions are allelic [9].

Reconstruction of teeth with dentinogenesis imperfecta is a difficult process [10]. Following the current procedures with composite materials does not guarantee a lasting restoration of the lost tissues [2, 5, 11]. Conventional adhesive systems are incapable of creating a true hybrid layer in the different histologi- 
cally structured dentin found in DI-II [12]. For this reason, we decided to carry out a morphologic study using scanning electron microscopy (SEM) to seek to understand more of the pathogenesis of this condition.

\section{Material and methods}

Four permanent first mandibular molars with a clinical diagnosis of dentinogenesis imperfecta type II were extracted from four patients aged 19-21 years on account of periapical lesions. Each tooth was placed in separate vials, numbered 1 to 4 . The patients had previously signed consent forms, and the study was approved by the Ethics Committee of the Jagiellonian University (KBET/174/B/2011).

The teeth were rinsed in distilled water, fixed, buffered, and stored in $4 \%$ glutaraldehyde. Next, they were dehydrated for 2 hours in an ethyl alcohol series, and finally dried in vacuum for 24 hours, in preparation for the next stage.

The teeth were embedded in a mixture of epoxy resin and carbon, and their surfaces were coated with gold in vacuum. Finally, the surfaces were prepared with polishing stones and cleansed with a stream of water before being examined in a Joel ISM 35CF scanning electron microscope (Joel, Tokyo, Japan).

\section{Results}

\section{Enamel}

In the sagittal sections, we noted multidirectional enamel rods with marked spaces between the prisms (Figure 1, sample 1). The crystalline forms of hydroxyapatite were clearly visible in these individual

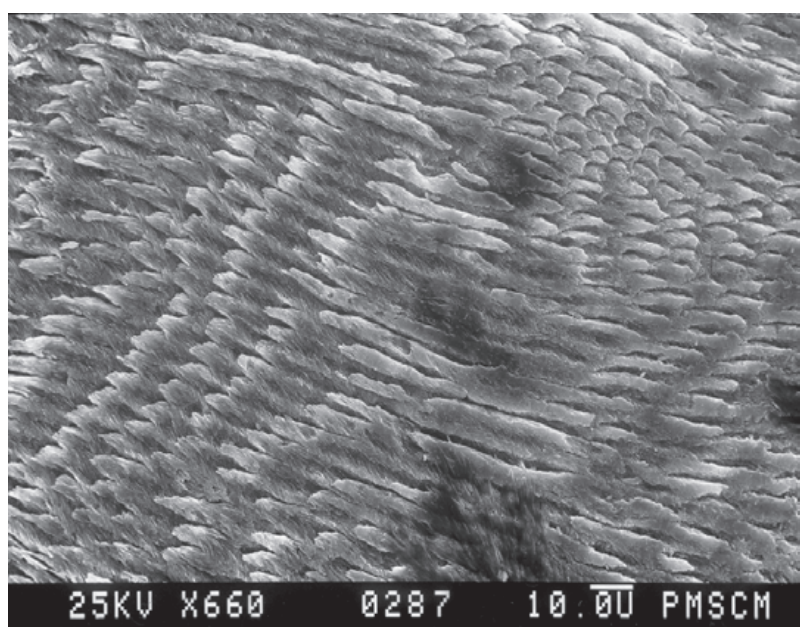

Figure 1. View in SEM of a cross-section of enamel (sample 1 ), with the prisms extending in different directions. Interprismatic spaces are clearly visible, as is the lack of major structural abnormalities. Magnification $660 \times$

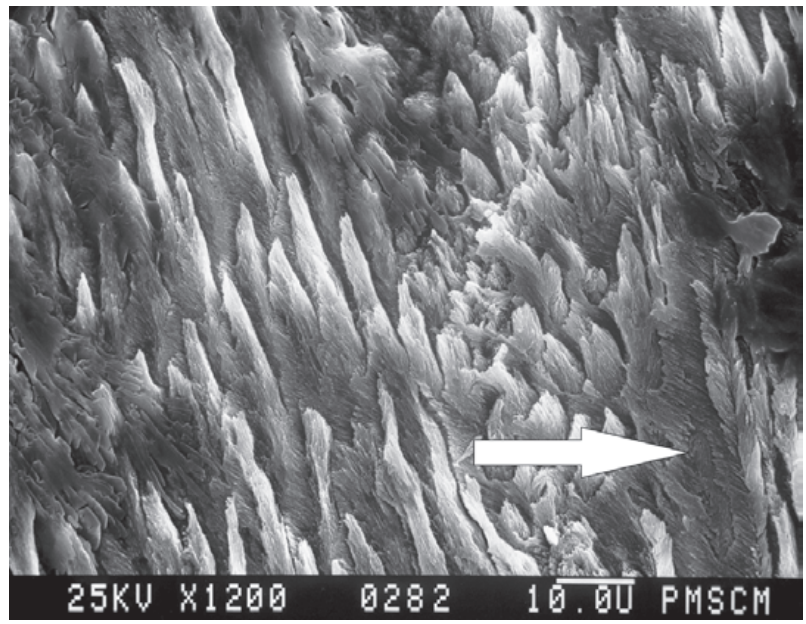

Figure 2. View in SEM of enamel prisms on the tooth surface (sample 1). The prisms have irregular shapes, and some crystalline forms are visible (indicated by the arrow). Magnification $1200 \times$

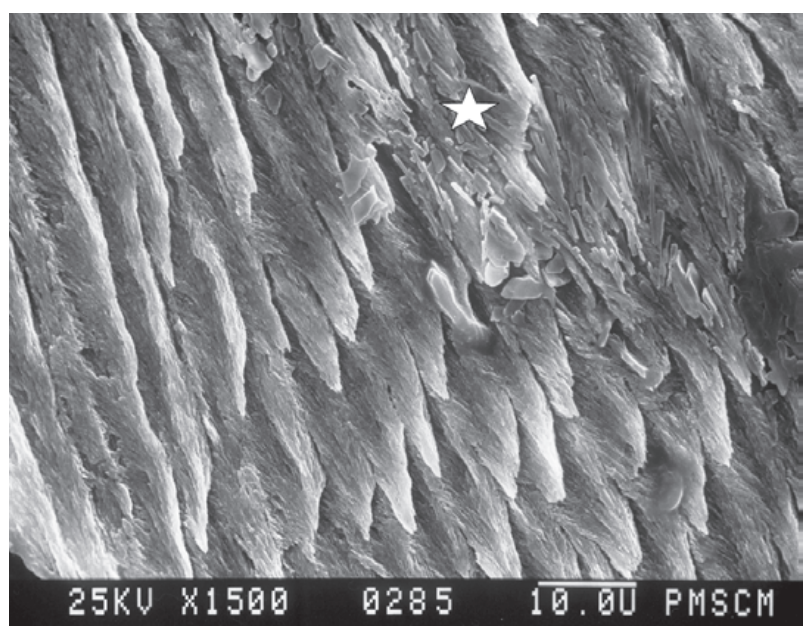

Figure 3. View in SEM of the enamel (sample 2). On the upper right of the exposed surface of the enamel, crystalline forms are visible (indicated by the asterisk), a result of lysis of the prisms. Magnification $1500 \times$

prisms (Figures 2 and 3, samples 1 and 2). The surface of the enamel was irregular, showing cracks and crevices. In some places granular remnants of the enamel were found, while in other parts of the enamel these were not observed.

\section{Dentin}

Our SEM examination of the DI-II teeth revealed the presence of many large dentinal tubules running in various directions, rather than the regular pattern normally seen (Figure 4, sample 3). These larger 


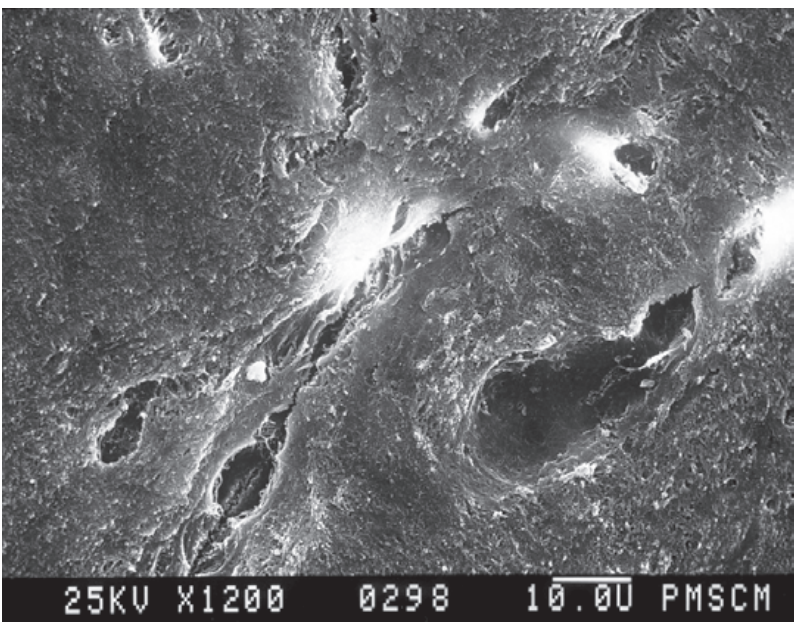

Figure 4. View in SEM of a sagittal section through dentin (sample 3). Large dentinal tubule cross-sections are visible, irregularly arranged. Magnification $1200 \times$

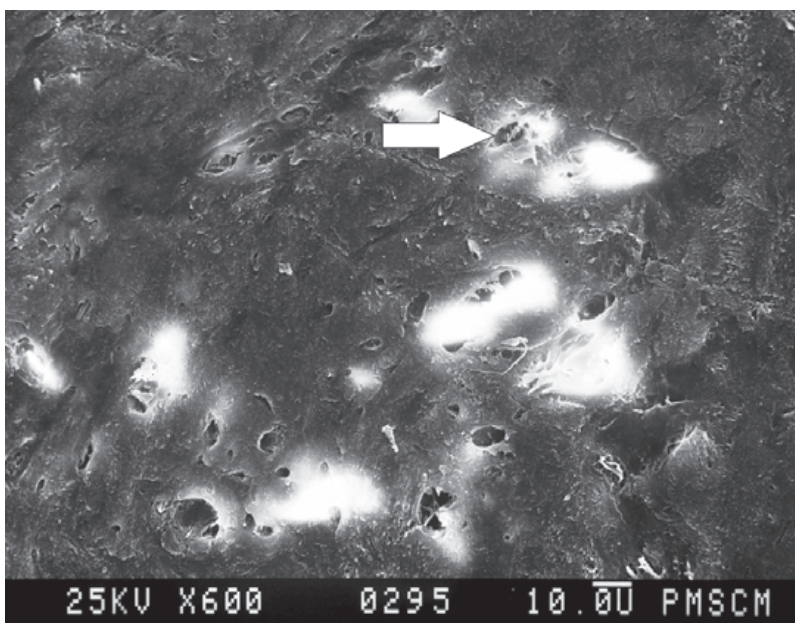

Figure 5. View in SEM of a sagittal section through dentin (sample 3). The small tubules contain odontoblastic processes and the larger show empty canals (indicated by the arrow). Magnification $600 \times$

tubules appeared devoid of odontoblastic processes, though these were visible in the smaller tubules (Figure 5, sample 3).

In a longitudinal section along one of the larger dentinal tubules, bundles of collagen fibers were observed to run in different directions along the walls (Figure 6, sample 4).

\section{Discussion}

In normal teeth, enamel is made up of prisms that extend from the dentino-enamel junction toward

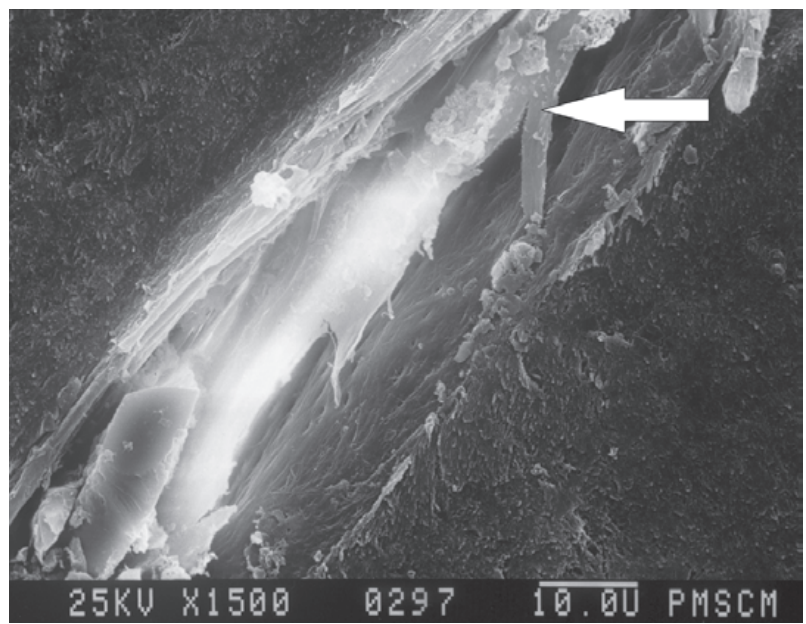

Figure 6. View in SEM of a longitudinal section of the dentin, showing a dental tubule. The inner wall of the tubule contains an odontoblastic process with its branches, which extend in different directions (indicated by the arrow). The process occupies almost the whole diameter of the tubule (sample 4). Magnification $1500 \times$

the outer surface of the enamel. These are arranged roughly perpendicularly to the dentino-enamel junction. Seldom are the prisms perfectly straight, and in some parts of the tooth they may display multiple curvatures. Each enamel rod appears to be encased in a rod sheath, and the sheathed prisms seem to be cemented together by an interrod substance [13]. The SEM images of sagittal tooth sections in the present study differed significantly: Firstly, within the enamel we observed enamel prism rods running in different directions, as well as marked interprismatic spaces. Hydroxyapatite crystals were clearly visible within the enamel prisms, which plainly showed a weakened tissue structure, thus implying a high susceptibility to adverse conditions in the mouth. Comparison of these descriptions reveals the structural differences between healthy enamel and the enamel in dentinogenesis imperfecta. Hall et al. showed structurally normal enamel in dentinogenesis imperfecta (but containing long and broad lamellae) and a normally scalloped dentino-enamel junction (DEJ) [14].

Secondly, in the healthy tooth, dentin is perforated by innumerable dentinal tubules that always contain odontoblastic processes. The tubules lie close together and extend from the pulp to the dentino-enamel junction. At their outer ends, the dentinal tubules are divided into a number of branches, while connecting adjacent dentinal tubules are often found along the length of the tubules [13].

Gallusi et al. [15], Salvolini et al. [8], De Coster et al. [16], Barron et al. [9], and Lindau et al. [17] have 
all reported irregular anomalies in the enamel-dentin junction. According to their observations, dentinal tubules are absent from large areas of the dentin; they also observed the presence of large channels in the dentin, just as we did in the present study. Gallusi et al. found that the dentin in dentinogenesis imperfecta showed an absence of tubules [15].

This disturbance of mineralization thus revealed is particularly significant in the enamel, since this is the tissue that covers the crown of the tooth. These anomalous structures of the hard dental tissues — visible with scanning electron microscopy — are formed during the development of the tooth, and subsequently become detrimental to the functional requirements of the tooth in the oral cavity, as well as to its resistance to bacterial, chemical, and physiological attack.

\section{Conclusions}

The histological and mineralization disturbances of the enamel and dentine, clearly revealed by this scanning electron microscopic examination, provide evidence of the structural defects that cause the problems of attrition and restoration loss found in patients with this genetic dental condition.

\section{Acknowledgments}

This study was supported by Grant K/ZDS/002455 of the Polish Ministry of Education and Research for the Collegium Medicum, Jagiellonian University.

Our sincere thanks are due to Professor Geoffrey Shaw for his English-language editing of this script.

Our sincere thanks also go to Professor Roman Pawlicki and Professor Zofia Knychalska-Karwan of the Collegium Medicum, Jagiellonian University, for their help in carrying out this study and in the preparation of the manuscript

The authors declare no potential conflicts of interest with respect to the authorship and/or the publication of this article.

\section{References}

1. Subramaniam P, Mathew S, Sugnani SN. Dentinogenesis imperfecta: a case report. J Indian Soc Pedod Prev Dent. 2008;26:85-87.
2. Shields ED, Bixler D, El-Kafrawy AM. A proposed classification for heritable human dentine defects with a description of a new entity. Arch Oral Biol. 1973;18:843-853.

3. Huth KC, Paschos E, Sagner T et al. Diagnostic features and pedodontic-orthodontic management in dentinogenesis imperfecta type II: A case report. Int J Paediatr Dent. 2002;12:316-321.

4. Miller WA, Winkler S, Rosenberg JJ et al. Dentinogenesis imperfecta traceable through five generations of a part American Indian family. Oral Surg Oral Med Oral Pathol. 1973;35:180-186.

5. Rivers JA, Staffanou RS. Restorative treatment of dentinogenesis imperfecta in a young adult. Compend Contin Educ Dent. 1985;6:548, 550, 552-544.

6. Pettiette MT, Wright JT, Trope M. Dentinogenesis imperfecta: endodontic implications. Case report. Oral Surg Oral Med Oral Pathol Oral Radiol Endod. 1998;86:733-737.

7. Bhandari S, Pannu K. Dentinogenesis imperfecta: a review and case report of a family over four generations. Indian J Dent Res. 2008;19:357-361.

8. Salvolini E, Di Giorgio R, Caselli E et al. Dentonogenesis imperfecta. Scanning electron microscopic study and microanalysis. Minerva Stomatol. 1999;48:87-92.

9. Barron MJ, McDonnell ST, Mackie I et al. Hereditary dentine disorders: dentinogenesis imperfecta and dentine dysplasia. Orphanet J Rare Dis. 2008;3:31.

10. Knezevic A, Tarle Z, Panduric V. Esthetic reconstruction of teeth in patient with dentinogenesis imperfecta - a case report. Coll Antropol. 2006;30:231-234.

11. Bouvier D, Duprez JP, Morrier JJ et al. Strategies for rehabilitation in the treatment of dentinogenesis imperfecta in a child: a clinical report. J Prosthet Dent. 1996;75:238-241.

12. Bouvier D, Leheis B, Duprez JP et al. Dentinogenesis imperfecta: long-term rehabilitation in a child.J Dent Child (Chic). 2008;75:192-196.

13. Permar D, Melfi R. Oral Embryology and Microscopic Anatomy. Philadelphia: Lea \& Febiger; 1977.

14. Hall RK, Maniere MC, Palamara J et al. Odontoblast dysfunction in osteogenesis imperfecta: an LM, SEM, and ultrastructural study. Connect Tis Res. 2002;43:401-405.

15. Gallusi G, Libonati A, Campanella V. SEM-morphology in dentinogenesis imperfecta type II: microscopic anatomy and efficacy of a dentine bonding system. Eur J Paediatr Dent. 2006;7:9-17.

16. De Coster PJ, Cornelissen M, De Paepe A et al. Abnormal dentin structure in two novel gene mutations [COL1A1. Arg134Cys] and [ADAMTS2, Trp795-to-ter] causing rare type I collagen disorders. Arch Oral Biol. 2007;52:101-109.

17. Lindau BM, Dietz W, Hoyer I et al. Morphology of dental enamel and dentine-enamel junction in osteogenesis imperfecta. Inter J Paediatr Dent. 2001;9:13-21.

Submitted: 25 August, 2013

Accepted after reviews: 26 August, 2013 